\title{
Copy number detection in discordant monozygotic twins of Congenital Diaphragmatic Hernia (CDH) and Esophageal Atresia (EA) cohorts
}

\author{
Danielle Veenma ${ }^{\star, 1,2}$, Erwin Brosens ${ }^{1,2}$, Elisabeth de Jong ${ }^{1,2}$, Cees van de Ven ${ }^{1}$, Connie Meeussen ${ }^{1}$, \\ Titia Cohen-Overbeek ${ }^{3}$, Marjan Boter ${ }^{2}$, Hubertus Eussen ${ }^{2}$, Hannie Douben ${ }^{2}$, Dick Tibboel ${ }^{1}$ and \\ Annelies de Klein ${ }^{2}$
}

The occurrence of phenotypic differences between monozygotic (MZ) twins is commonly attributed to environmental factors, assuming that MZ twins have a complete identical genetic make-up. Yet, recently several lines of evidence showed that both genetic and epigenetic factors could have a role in phenotypic discordance after all. A high occurrence of copy number variation (CNV) differences was observed within MZ twin pairs discordant for Parkinson's disease, thereby stressing on the importance of post-zygotic mutations as disease-predisposing events. In this study, the prevalence of discrepant CNVs was analyzed in discordant MZ twins of the Esophageal Atresia (EA) and Congenital Diaphragmatic Hernia (CDH) cohort in the Netherlands. Blood-derived DNA from 11 pairs (7 EA and $4 \mathrm{CDH}$ ) was screened using high-resolution SNP arrays. Results showed an identical copy number profile in each twin pair. Mosaic chromosome gain or losses could not be detected either with a detection threshold of $20 \%$. Some of the germ-line structural events demonstrated in five out of eleven twin pairs could function as a susceptible genetic background. For example, the 177-Kb loss of chromosome 10q26 in CDH pair-3 harbors the TCF7L2 gene (Tcf4 protein), which is implicated in the regulation of muscle fiber type development and maturation. In conclusion, discrepant CNVs are not a common cause of twin discordancy in these investigated congenital anomaly cohorts.

European Journal of Human Genetics (2012) 20, 298-304; doi:10.1038/ejhg.2011.194; published online 9 November 2011

Keywords: monozygotic twins; copy number variation; congenital anomaly; phenotypic discordance; congenital diaphragmatic hernia; esophageal atresia

\section{INTRODUCTION}

Monozygotic (MZ) twin comparisons have been used for many decades to specify contributions of both nature (heredity) and nurture (environment). ${ }^{1}$ Normally, the study design is based on the presumption that $\mathrm{MZ}$ twins come from one fertilized egg, and therefore have complete identical genetic make-ups. Yet, recently several lines of evidence suggested that genetic and epigenetic factors could have a role in $\mathrm{MZ}$ phenotypic variances after all. ${ }^{2-6}$ Using a BAC array platform, Bruder et $a l^{6}$ demonstrated that discordance in their MZ Parkinson's disease (PD) twin cohort of nine individuals could be the result of copy number variation $(\mathrm{CNV})$ differences. However, Baranzini et $a l^{7}$ could not reproduce this high intra-twin pair variability of structural variants using both array and next-generation sequencing in three twin pairs discordant for Multiple Sclerosis.

We investigated whether discrepant CNVs could cause discordance in MZ twin pairs of the Dutch Esophageal Atresia (EA (MIM 189960)) and Congenital Diaphragmatic Hernia (CDH (MIM 142340)) cohort. Blood-derived DNA from 11 (7 EA and $4 \mathrm{CDH}$ ) pairs of $\mathrm{MZ}$ twins was screened using high-resolution SNP arrays.

EA generally presents at birth with a defective formation of the esophagus with or without a fistulous tract to the trachea. Although not lethal in most cases, long-term morbidity has a significant role in these patients. $\mathrm{CDH}$ is a more severe birth defect characterized by defective formation of the diaphragm, lung hypoplasia and pulmonary hypertension. Despite medical advances mortality for isolated cases is $20 \%$ and for none-isolated cases up to $60 \%$. Both EA and CDH are presumed to have a multifactorial etiology and the identification of chromosomal aberrations and knockout animal models provide strong evidence for a genetic component. ${ }^{8}$ In contrast, both anomalies present with low twin concordance rates, $10.7 \%$ and $15.6 \%$ for EA and $\mathrm{CDH}$, respectively, and sibling recurrence rates are low (1-2\%) as well. Shaw-Smith ${ }^{9}$ already pointed out that the incidence of twinning in EA is 2.6 times higher than statistically expected. In all, 206 pairs are described in the literature up until now; however, information on zygosity is less thorough. ${ }^{9-16}$ Orford et al ${ }^{15}$ stated that at least $80 \%$ of reported EA twins are same-sex pairs. In total, 22 out of these 206 twin pairs are concordant for the EA phenotype. In the literature, 77 twin pairs have been described for $\mathrm{CDH}$ of which 53 were recognized as MZ. ${ }^{17-20}$ Twelve pairs were concordant for the $\mathrm{CDH}$ phenotype.

The rationale of this study was to investigate whether CNVs in the affected twin sibling could account for phenotypic discordance of either EA or CDH MZ twin siblings. Although results showed no such

${ }^{1}$ Department of Paediatric Surgery, Rotterdam, The Netherlands; ${ }^{2}$ Department of Clinical Genetics, Rotterdam, The Netherlands; ${ }^{3}$ Department of Obstetrics and Gynaecology, Erasmus MC-Sophia, Rotterdam, The Netherlands

${ }^{*}$ Correspondence: Dr D Veenma, Department of Paediatric Surgery, Dr. Molewaterplein 50, Rm SK-3286, PO Box 2040,3000 CA Rotterdam, The Netherlands. Tel: +31 10 7043492; Fax: +31 10 7044736; E-mail: d.veenma@erasmusmc.nl

Received 30 May 2011; revised 9 September 2011; accepted 15 September 2011; published online 9 November 2011 
Table 1a Clinical features EA cohort

\begin{tabular}{|c|c|c|c|c|c|c|c|}
\hline \multirow[b]{2}{*}{ Pair EA } & \multirow[b]{2}{*}{ GA (weeks) } & \multicolumn{2}{|c|}{ Birth order } & \multirow[b]{2}{*}{ Obstetric history } & \multirow[b]{2}{*}{$E A$} & \multirow[b]{2}{*}{ Fistel } & \multirow[b]{2}{*}{ Type of additional anomalies } \\
\hline & & (Patient) & (Healthy twin) & & & & \\
\hline EA1 & 37.3 & 1 & 2 & Breech presentation & + & + & $\begin{array}{l}\text { Dysmorphic } \\
\text { Auricular tags, Cleft uvula } \\
\text { Abnormal dermatoglyphics } \\
\text { Heart } \\
\text { ASD } \\
\text { Lung } \\
\text { Lung hypoplasia right } \\
\text { Neurologic/skeletal } \\
\text { Scoliosis } \\
\text { Fusion of vertebrae } \\
\text { Hemivertebrae } \\
\text { IUGR }\end{array}$ \\
\hline EA2 & 36 & 2 & 1 & $\begin{array}{l}\text { Breech presentation } \\
\text { Maternal medication: } \\
\text { Sintrom }\end{array}$ & + & + & $\begin{array}{l}\text { Heart } \\
\text { VSD } \\
\text { Lung } \\
\text { Lunghypoplasia }\end{array}$ \\
\hline EA4 & NA & NA & NA & NA & + & - & - \\
\hline EA5 & 33.5 & 1 & 2 & $\begin{array}{l}\text { Breech presentation } \\
\text { Fever durante partu }\end{array}$ & + & + & $\begin{array}{l}\text { Dysmorphic } \\
\text { Triangular face, deep set eyes, } \\
\text { Palpebral fissures slant down } \\
\text { Small mandible } \\
\text { Thin fingers, hypoplastic thumbs } \\
\text { Proximal placement of thumb } \\
\text { Hypoplastic or absent radii } \\
\text { Sacral hemangioma healthy twin }\end{array}$ \\
\hline
\end{tabular}

Table 1b Clinical features $\mathrm{CDH}$ cohort

\begin{tabular}{|c|c|c|c|c|c|c|}
\hline \multirow[b]{2}{*}{ Pair $\mathrm{CDH}$} & \multirow[b]{2}{*}{ GA (weeks) } & \multicolumn{2}{|c|}{ Birth order } & \multirow[b]{2}{*}{ Obstetric history } & \multirow[b]{2}{*}{$C D H$} & \multirow[b]{2}{*}{ Type of additional anomalies } \\
\hline & & (Patient) & (Healthy twin) & & & \\
\hline $\mathrm{CDH} 1$ & 35.3 & 2 & 1 & $\begin{array}{l}\text { Sectio Caesarea } \\
\text { Breech presentation }\end{array}$ & Left & - \\
\hline $\mathrm{CDH} 2$ & 33.4 & 1 & 2 & $\begin{array}{l}\text { Sectio Caesarea } \\
>24 \mathrm{~h} \text { ruptured membrane }\end{array}$ & Left & - \\
\hline $\mathrm{CDH} 3$ & 38.5 & 2 & 1 & - & Right & $\begin{array}{l}\text { Urogenital } \\
\text { Inguinal hernia } \\
\text { Hydrocele testis }\end{array}$ \\
\hline $\mathrm{CDH} 4$ & 34.1 & 2 & 1 & Sectio Caesarea & Left & $\begin{array}{l}\text { Dysmorphic } \\
\text { Small mandibula } \\
\text { IUGR }\end{array}$ \\
\hline
\end{tabular}

Abbreviations: ASD, Atrial Septal defect; CDH, Congenital Diaphragmatic Hernia; EA, Esophageal Atresia; GA, gestational age; IUGR, Intra Uterine Growth Retardation; VSD, Ventrical Septal Defect. Unfortunately, for a few EA subjects detailed clinical data are unavailable.

proof, germ-line structural events were detected and these could represent a susceptible genetic background as seen in other genetic anomalies. Results are discussed in the context of earlier MZ twin reports.

\section{MATERIALS AND METHODS}

\section{Ethics statement}

Research involving human participants has been approved by the Medical Ethical Committee (METC) at Erasmus MC, which specifically approved for blood 
withdrawal of both twins and their parents. Informed consent forms were obtained for the index case and his/her parents at once and for the healthy twin separately.

\section{Patients}

The 11 affected twin samples were collected from the congenital anomaly cohort in Rotterdam (Erasmus MC Sophia's Hospital, The Netherlands) in which $541 \mathrm{EA}$ and $626 \mathrm{CDH}$ patients are currently registered. Of these, $22 \mathrm{CDH}$ patients ( 14 dizygotic, $5 \mathrm{MZ}$, and 3 not tested) and 35 EA patients (6 dizygotic, $9 \mathrm{MZ}$, and 20 not tested) were the result of a twin pregnancy. Included were those twin samples with a written parental informed consent, quality material of both siblings and confirmed monozygosity by STR profiling (AmpFISTR identifiler PCR amplification kit, Applied Biosystems, Foster City, CA, USA). Another exclusion criterion was the identification of a genetic abnormality, most commonly an aneuploidy.

\section{DNA isolation}

Automated DNA extraction from peripheral blood (or skin fibroblasts in case of two affected CDH twins) was performed using local standard protocols. DNA quality and concentration were checked with the Quant-iT PicoGreen dsDNA Kit (Invitrogen Corporation, Carlsbad, CA, USA).

\section{Whole-genome high-resolution SNP array}

SNP analysis was carried out using the Illumina HumanCytoSNP-12 version 2.2. (Illumina, San Diego, CA, USA). This chip includes 220000 of the most informative SNPs markers with a median physical distance of $6.2 \mathrm{~Kb}$. DNA samples were processed according to the manufacturer's protocol. The call rate of this array batch was above 0.98 , except for one sample.

\section{SNP array analysis}

Data for each bead chip were self-normalized in Genomestudio GT (Illumina) using information contained within the array. Copy number estimates for each individual sample were determined by comparison with a common reference set of 200 samples from the HapMAP project (www.hapmap.org/downloads/raw_data) supplied by Illumina (Manifest files) and visualized in the Nexus software program (version five, Biodiscovery, El Segundo, CA, USA) as $\log 2$ ratios. Analysis settings were as follow: both SNP-FASST and SNP-Rank segmentation methods were executed independently with significance thresholds ranging from $1 \times 10^{-4}$ to $1 \times 10^{-6}$ and log-ratio thresholds of 0.18 and -0.18 for duplication and deletions, respectively. The max contiguous probe spacing was $1000 \mathrm{~Kb}$ and the minimum number of probes per segment was set to three, limiting CNV detection to sizes $>18.6 \mathrm{~Kb}$. Subsequently, only $\mathrm{CNVs}>50 \mathrm{~Kb}$ were validated. Paired analysis for deletions and duplications was performed in each affected twin versus its healthy co-twin. As described recently, high-resolution (SNP) arrays are suitable for detection of both germ-line and mosaic CNVs. ${ }^{21-25}$ Mosaic copy number aberrations are hallmarked by a concomitant change of log2 intensity signal and a shift in b-allele frequency. The detection limit (sensitivity) of the Nexus SNP-FASST algorithm for mosaic CNVs is $20 \%$ using a heterozygous imbalance threshold of $0.45 .{ }^{22}$ To review functionality of each putative $\mathrm{CNV}$ at once, occurrence frequencies in a qualified normal pediatric cohort of 2026 individuals $^{26}$ (CHOP; http://cnv.chop.edu/) and in the DGV (http:// www.tcag.ca) were uploaded in the Nexus program as well. Since these populations display various ethnic backgrounds, comparison with an in-house normal reference set was performed as well. Additionally, possible intra-twin pair genotype differences (with respect to all SNP markers presented on the array) were evaluated in Genomestudio GT using the paired analysis settings.

\section{Validation using fluorescent in-situ hybridization and relative-quantitative $P C R$ analysis}

Confirmation of each CNV with quantitative real-time PCR and/or FISH was executed in the twin siblings and their parents according to local standard protocols with minor modifications. ${ }^{22,27}$ For FISH, BAC clones were selected from the UCSC genome browser (http://genome.ucsc.edu/), purchased at BACPAC resources center (Oakland, CA, USA) and labelled (Random Prime labelling system; Invitrogen Corporation) with Bio-16-dUTP or Dig-11-dUTP (Roche Applied Science, Indianapolis, IN, USA). After validation on control metaphases, the chromosome 22 BAC clones RP11-62K15 and RP1-66M5 were used for confirmation in EA pair-I.

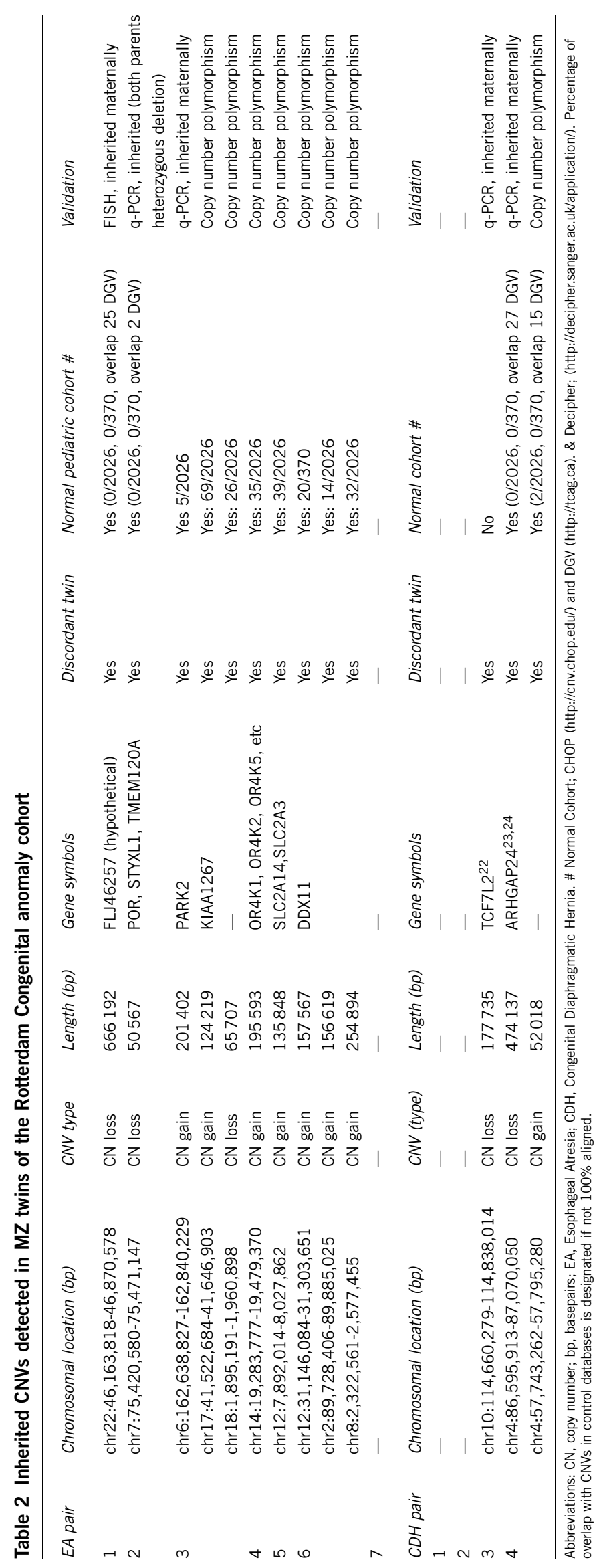


Primer pairs for quantitative real-time PCR were designed from unique sequences within the minimal deleted or duplicated regions of each copy number change using Primer Express software v2.0 (Applied Biosystems, Carlsbad, CA, USA). The nucleotide BLAST algorithm at NCBI (http:// www.ncbi.nlm.nih.gov/BLAST/) was used to confirm that each PCR amplification product was unique. Quantitative PCR analyses were performed using a LightCycler 1.5 instrument in combination with LightCycler FastStart DNA Master SYBR Green I kits (Roche Molecular Diagnostics, Indianapolis, IN, USA). Experiments were designed with a region of the C14ORF145 gene serving as a control locus as previously described. ${ }^{27}$

\section{RESULTS}

Clinical characterization and monozygosity screening of twin pairs Clinical features of each twin pair are summarized in Table 1. Briefly, 7 out of 11 pairs were discordant for the phenotype of EA (Table 1a) and 4 out of 11 for CDH (Table 1b). Four out of eleven EA-affected patients harbored (major) additional anomalies. Considering $\mathrm{CDH}$, there is a variable expression of left and right $\mathrm{CDH}$ with all persons (as expected) featuring lung hypoplasia. We are dealing with an isolated $\mathrm{CDH}$ cohort since most anomalies in pairs 3 and 4 are minor. Finally, zygosity status of each twin pair was confirmed (data not shown) by STR profiling using the commercially available STR identifiler kits of Applied Biosystems.

\section{Paired CNV analysis of discordant MZ twins}

Results of the paired $\mathrm{CNV}$ analysis of each $\mathrm{MZ}$ twin couple are summarized in Table 2 showing no evidence of pathogenic CNV discordance in both congenital anomaly cohorts. In order to detect mosaic (somatic) aberrations, specific attention was payed to b-allele frequency changes as well. In the EA cohort, a total of 10 germ-line CNVs were identified. Seven concerned common copy number

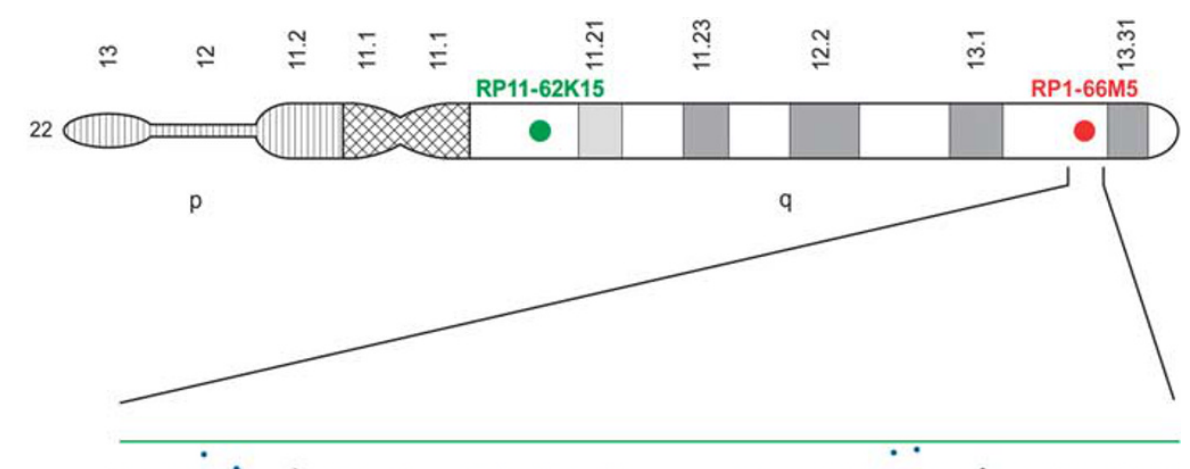

EA 1-1
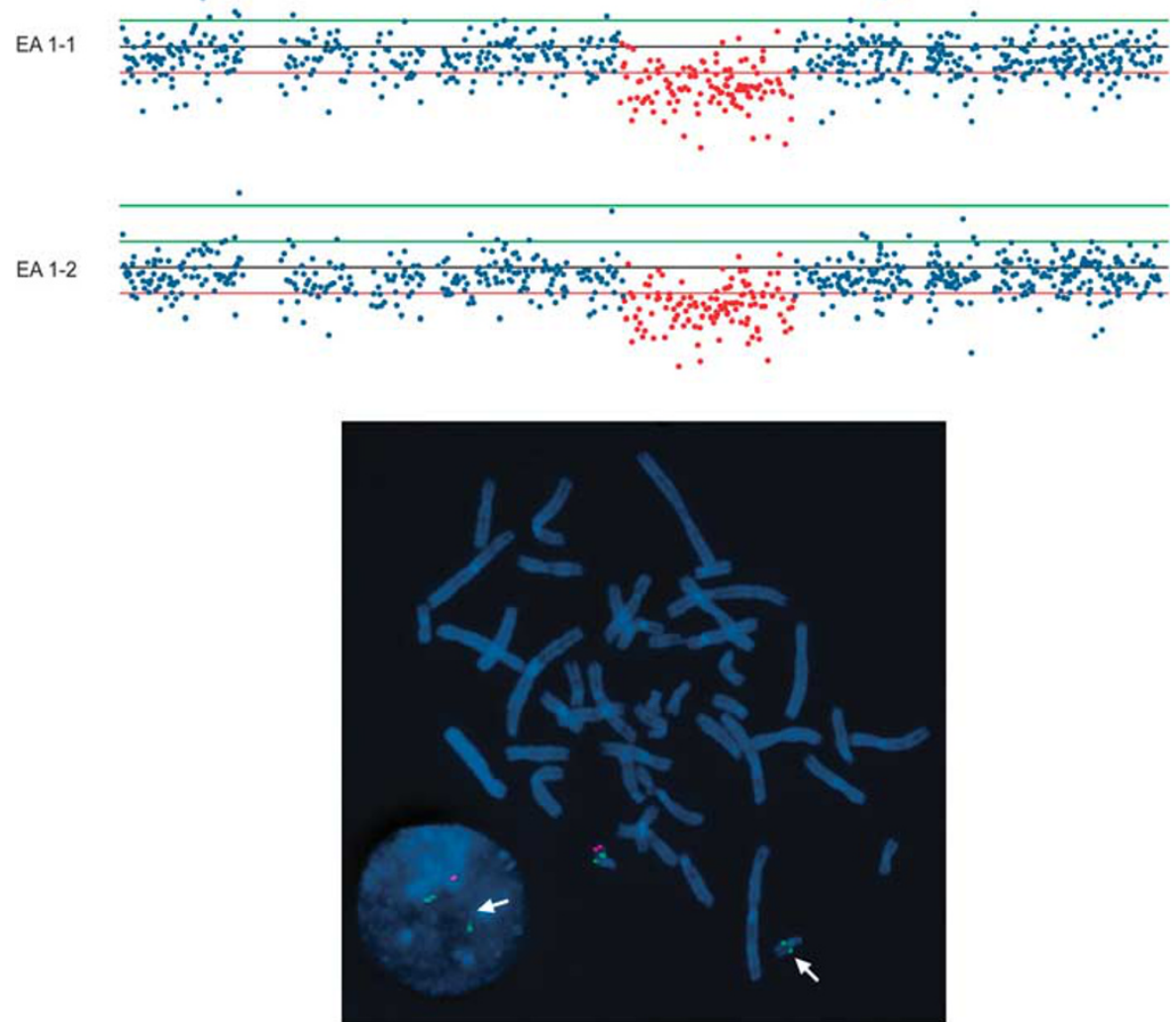

Figure $1 \mathrm{SNP}$ and fluorescent in-situ hybridization results of inherited chromosome 22 CNV in EA pair-I. Nexus results (Top) of the 666-Kb deletion on chromosome 22q13.3 in both individuals of EA pair-I showing a clear drop in log2 intensity signal validated by FISH (Bottom) on metaphase chromosomes of the affected EA twin-1. Probes are control: RP11-62K15 (green) and target: RP11-66M5 (red). Parental analysis (results not shown) demonstrated that this genomic event is inherited from the mother and therefore less likely pathogenic. In addition, no gene is allocated to this region neither are any miRNA transcripts hampering the identification of functional elements in this region as well. 
polymorphisms defined by the occurrence of the CNV in at least five individuals of qualified normal pediatric cohorts in the literature. The remaining three events were present in both the twin and at least one healthy parent, and are therefore less likely to be pathogenic as well. For example, the $666-\mathrm{Kb}$ sized chromosome 22q deletion in EA pair-1 (Figure 1) was found both in the healthy twin and his mother and partly overlaps with CNVs cataloged in control cohorts.

Existence of inherited CNVs was detected in the $\mathrm{CDH}$ cohort as well. A total of three CNVs were distinguished of which two are not prevalent in normal cohorts. All three events were present in the healthy twin as well. Figure 2 represents the $177-\mathrm{Kb}$ loss of chromosome 10q26 in CDH pair-3 and harbors the TCF7L2 gene (Tcf4 protein), which is mainly known for its involvement in blood glucose homeostasis as a result of Wnt signalling changes.

Not ruled out in this study are the presence of balanced genomic alterations and small $(<50 \mathrm{~Kb})$ or very-low mosaic $(<20 \%)$ chromo- some aberrations beyond the detection level of our experimental approach.

\section{SNP genotype analysis MZ twin cohorts}

SNP genotype differences between the affected and unaffected twin siblings were evaluated for each SNP on the Illumina bead chip. After removal of less accurately called SNPs, genotyping analysis showed concordance for almost all SNPs $(n=299671)$ within each MZ pair. A total of five SNPs in three EA pairs were dissimilar and three SNPs in two $\mathrm{CDH}$ pairs (Table 3). CDH pair-3 showed discrepancy for 99 SNPs, which could be attributed to less overall genotyping accuracy and therefore was not analyzed further. Until now, only rs2824374 (which is closely linked to the CXADR gene) could be associated with embryonic (mal) development, however, literature only reports on effects to the kidneys and cochlea. ${ }^{28,29}$ None of the other identified discordant intra-twin SNP loci are directly linked to a phenotype.
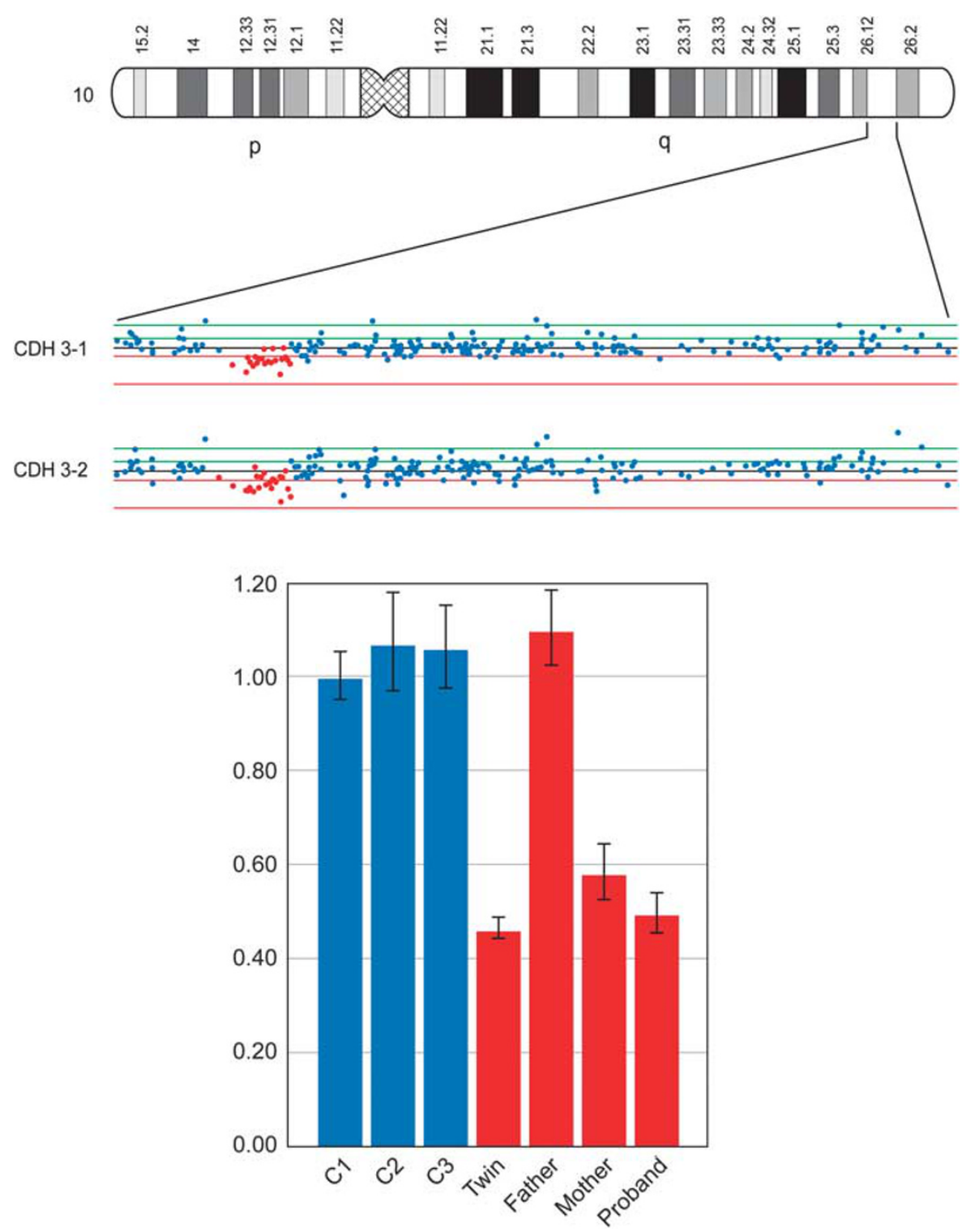

Figure 2 SNP and RT-PCR results of inherited chromosome 10-CNV in CDH pair-3. Nexus result (TOP) of the chromosome 10q26 deletion event in CDH pair-3 showing a clear drop in log2 intensity signal which was confirmed by relative q-PCR (Bottom) in the affected proband, the unaffected twin and the mother. 
Table 3 Discordant SNPs in MZ twin pairs of the Rotterdam Congenital anomaly cohort

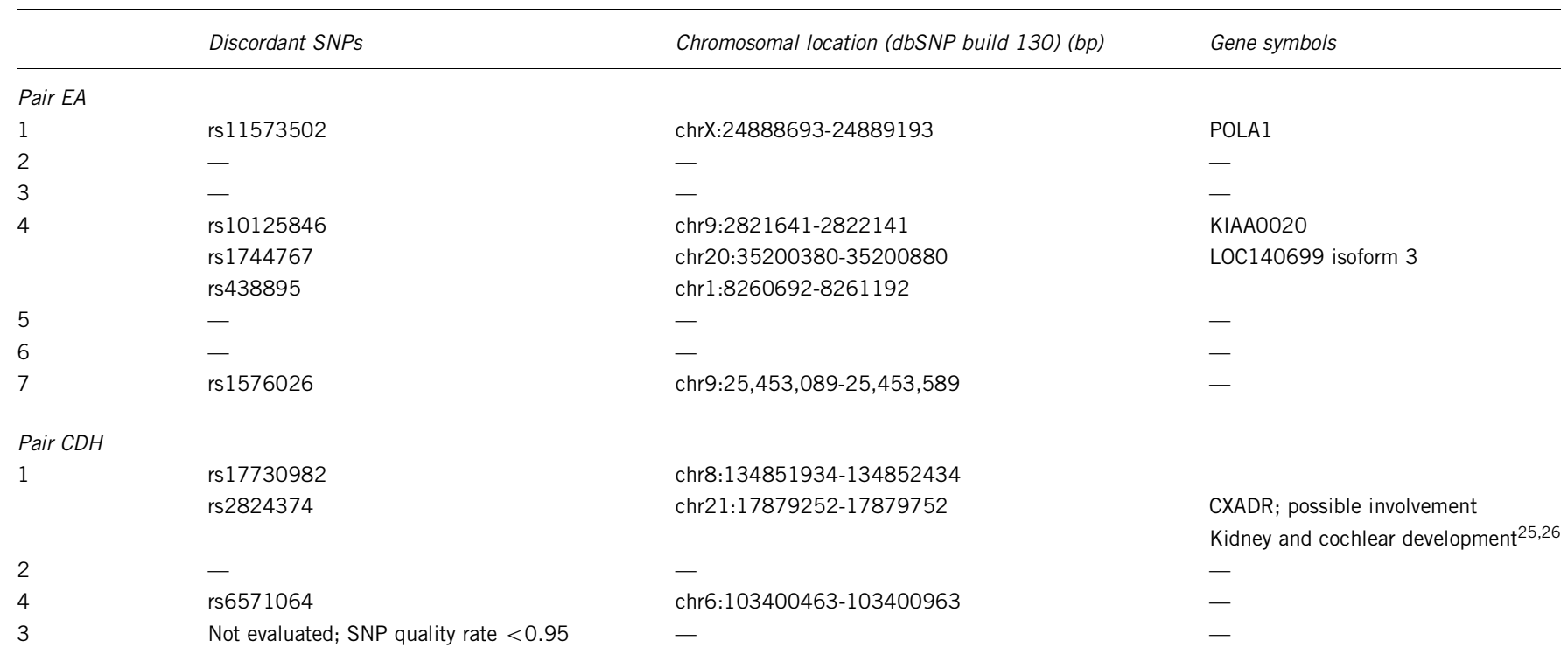

Abbreviations: bp, basepairs; EA, Esophageal Atresia; CDH, Congenital Diaphragmatic Hernia.

\section{DISCUSSION}

A high occurrence of copy number variants that differed between siblings discordant for PD was recently suggested. ${ }^{6}$ However, intra-twin pair variability for germ-line CNVs could not be detected in our subset of EA and CDH MZ twins. Within the limitations of the used experimental approach, structural variants in mosaic form (above 20\%) could neither be demonstrated. Application of nextgeneration sequencing methods will allow for an easier and more sensitive calling of the smallest mosaic aberrations in the near future and will add up to the (scarce) data generated recently on this topic by some other groups. ${ }^{30-33}$

Various causes could account for the discrepancy in CNV findings between our congenital anomaly twin cohort and the Parkinson's cohort. First of all, an age factor: the rather high prevalence of mosaic CNVs in PD twins could have been generated during lifetime. This was suggested by a small study of the group of Dumanski et al, ${ }^{34,35}$ who identified mosaic aberrations in a wide range of tissues of three phenotypically normal individuals. This hypothesis would imply that age-accumulated (tissue-specific) $\mathrm{CNV}$ events could have a role in diseases developing symptoms later in life. Consequently, they are expected to contribute less to congenital disorders. Second, differences in CNV prevalence between our study and the Parkinson's study could be based on methodological differences such as choice of platform. Although Bruder et al presented confirmative evidence for a few of their CNVs using a different platform, detailed confirmation of most $\mathrm{CNV}$ s was lacking. On the other hand, structural DNA variation might have a minor role in EA and $\mathrm{CDH}$ pathophysiology, suggesting that in these congenital cohorts the focus should be widened on environmental and epigenetic factors. Two recent studies ${ }^{2,7}$ revealed a (significant) proportion of epigenetic variability between $\mathrm{MZ}$ twins in investigated tissues. However, in the Multiple Sclerosis twin cohort study these changes could not account for disease discordance. A similar study for EA, CDH, or other congenital anomalies is difficult to perform, since the target tissues cannot be obtained from the healthy co-twin for obvious reasons. Structural variations restricted to the affected esophagus and diaphragm tissue could represent another cause for twin discordancy, yet was not excluded in this MZ cohort due to unavailability of the affected material.
Finally, although our results showed no proof for CNV contribution to phenotypic MZ discordance, germ-line structural events were detected in both cohorts and these events could represent a so-called susceptible genetic background. In five out of eleven twin pairs, germline $\mathrm{CNVs}$ were identified. These were rarely detected in a specific pediatric normal population ${ }^{26}$ and/or our in-house control cohort and could therefore represent an increased susceptibility to congenital anomalies by means of a dosage responsive or position effect. For example, the 177-Kb loss of chromosome 10q26 in $\mathrm{CDH}$ pair-3 might be of functional importance. A recent report demonstrated Tcf4 (alias $T c f 7 l 2$ ) expression in connective tissue fibroblasts during development and suggested its role in the regulation of muscle fiber type development and maturation. ${ }^{36}$ Additionally, certain polymorphisms and mutations in TCF7L2 are linked to an increased risk of type 2 diabetes. ${ }^{37}$ This implies that loss of one functional TCF7L2 allele might be associated with (super) normal glucose tolerance. Indeed, we observed evidence of increased serum glucose (a glucose of $12.2 \mathrm{mmol} / \mathrm{l}$ was identified within $24 \mathrm{~h}$ postnatal) in the affected individual of twin pair-3. However, also one normal glucose level (glucose $3.6 \mathrm{mmol} / \mathrm{l}$ ) was determined within the same time window and since this patient was critically ill and died shortly thereafter no absolute conclusions can be drawn from these results. The healthy twin had an unremarkable medical record so far. Similarly, the haploinsufficient ARHGAP24 gene in $\mathrm{CDH}$ pair-4 (encoding a vascular, cell-specific GTPase-activating protein) could confer genetic susceptibility for $\mathrm{CDH}$ by means of its function in modulating angiogenesis and through its interaction with filamin-A. ${ }^{38,39}$ Girirajan et $a l^{40}$ recently demonstrated that a second hit may elicit a severe phenotype in offspring of healthy CNV carriers. Hypothetically, this second hit can constitute another CNV in the same or associated disease pathway as well as a pathogenic SNP. These results underline the importance of archiving all genomic events (also those with a 'benign' nature at first sight) in a freely accessible database such as initiated by the ISCA consortium (https://www.iscaconsortium.org). Detailed and unbiased phenotyping is crucial for the understanding of the more complex genotype-phenotype correlations as well.

In summary, we investigated whether the existence of discrepant $\mathrm{CNV}$ s could be causal to the phenotypic discordance in $\mathrm{MZ}$ twin pairs 
of the EA and CDH cohort in Rotterdam and found no such proof. Prospective collection of DNA material in various MZ twin cohorts is warranted to evaluate the possibility of such genetic factors contributing to human phenotypic variability in general and to twin discordance specific. We feel that the use of high-resolution SNP arrays and sequencing-based methods are more suitable in these designs than BAC arrays. Finally, phenotypic correlations can only be made after proper analysis in normal cohorts as well.

\section{CONFLICT OF INTEREST}

The authors declare no conflict of interest.

\section{ACKNOWLEDGEMENTS}

We would like to thank all patients, specifically all twins and their parents for making this study possible. Also, we thank Tom de Vries-Lentsch for his help in preparing the figures. This study was funded by the Sophia's Children's Hospital Foundation for Medical Research, Rotterdam, The Netherlands (SSWO 493 and 551). The funders had no role in study design, data collection and analysis, decision to publish, or preparation of the manuscript.

1 Hall JG: Twinning. Lancet 2003; 362: 735-743.

2 Kaminsky ZA, Tang T, Wang SC et al: DNA methylation profiles in monozygotic and dizygotic twins. Nat Genet 2009; 41: 240-245.

3 Kimani JW, Shi M, Daack-Hirsch S et al: X-chromosome inactivation patterns in monozygotic twins and sib pairs discordant for nonsyndromic cleft lip and/or palate. Am J Med Genet 2007; 143A: 3267-3272.

4 Machin GA: Some causes of genotypic and phenotypic discordance in monozygotic twin pairs. Am J Med Genet 1996; 61: 216-228.

5 Streit A, Sommer RJ: Genetics: random expression goes binary. Nature 2010; 463 : 891-892.

6 Bruder CE, Piotrowski A, Gijsbers AA et al: Phenotypically concordant and discordant monozygotic twins display different DNA copy-number-variation profiles. Am J Human Genet 2008; 82: 763-771.

7 Baranzini SE, Mudge J, van Velkinburgh JC et al: Genome, epigenome and RNA sequences of monozygotic twins discordant for multiple sclerosis. Nature 2010; 464: 1351-1356

8 Holder AM, Klaassens M, Tibboel D, de Klein A, Lee B, Scott DA: Genetic factors in congenital diaphragmatic hernia. Am J Human Genet 2007; 80: 825-845.

9 Shaw-Smith C: Oesophageal atresia, tracheo-oesophageal fistula, and the VACTERL association: review of genetics and epidemiology. J Med Genet 2006; 43: 545-554.

10 Sunagawa S, Kikuchi A, Yoshida S et al: Dichorionic twin fetuses with VACTERL association. J Obstet Gynaecol Res 2007; 33: 570-573.

11 Depaepe A, Dolk H, Lechat MF: The epidemiology of tracheo-oesophageal fistula and oesophageal atresia in Europe. EUROCAT Working Group. Arch Dis Child 1993; 68 : 743-748.

12 Farquhar J, Carachi R, Raine PA: Twins with oesophageal atresia and the CHARGE association. Eur J Pediatr Surg 2002; 12: 56-58.

13 Khan RA, Narashimhan KL: Discordance in twins for esophageal atresia with tracheoesophageal fistula. Indian J Pediatr 2009; 76: 334-335.

14 Ohno K, Nakamura T, Azuma T et al: Esophageal atresia with tracheoesophageal fistula in both members of monozygotic twins. Pediatr Surg Int 2008; 24: 1137-1139.

15 Orford J, Glasson M, Beasley S, Shi E, Myers N, Cass D: Oesophageal atresia in twins. Pediatr Surg Int 2000; 16: 541-545.
16 Trobs RB, Knupffer M, Schutz A, Cernaianu G, Hirsch W, Regenthal R: Congenital oesophageal atresia discordant for tracheo-oesophageal fistula occurring in a set of dizygotic twins. Eur J Pediatr Surg 2006; 16: 260-264.

17 Donahoe PK, Noonan KM, Lage K: Genetic tools and algorithms for gene discovery in major congenital anomalies. Birth Defects Res 2009; 85: 6-12.

18 Lau ST, Kim SS, Ledbetter DJ, Healey PJ: Fraternal twins with Morgagni hernias. J Pediatr Surg 2005; 40: 725-727.

19 Machado AP, Ramalho C, Portugal R et al: Concordance for bilateral congenital diaphragmatic hernia in a monozygotic dichorionic twin pair - first clinical report. Fetal Diagn Ther 2010; 27: 106-109.

20 Pober BR, Lin A, Russell M et al: Infants with Bochdalek diaphragmatic hernia: sibling precurrence and monozygotic twin discordance in a hospital-based malformation surveillance program. Am J Med Genet 2005; 138A: 81-88.

21 Conlin LK, Thiel BD, Bonnemann CG et al: Mechanisms of mosaicism, chimerism and uniparental disomy identified by single nucleotide polymorphism array analysis. Hum Mol Genet 2010; 19: 1263-1275.

22 Veenma D, Beurskens $\mathrm{N}$, Douben $\mathrm{H}$ et al: Comparable low-level mosaicism in affected and non affected tissue of a complex CDH patient. PLoS One 2010; 5: e15348.

23 Shaffer LG, Kashork CD, Saleki R et al: Targeted genomic microarray analysis for identification of chromosome abnormalities in 1500 consecutive clinical cases. J Pediatr 2006; 149: 98-102.

24 Ballif BC, Rorem EA, Sundin K et al: Detection of low-level mosaicism by array CGH in routine diagnostic specimens. Am J Med Genet A 2006; 140: 2757-2767.

25 Xiang B, Li A, Valentin D, Nowak NJ, Zhao H, Li P: Analytical and clinical validity of whole-genome oligonucleotide array comparative genomic hybridization for pediatric patients with mental retardation and developmental delay. Am J Med Genet A 2008; 146A: 1942-1954.

26 Shaikh TH, Gai X, Perin JC et al: High-resolution mapping and analysis of copy number variations in the human genome: a data resource for clinical and research applications. Genome Res 2009; 19: 1682-1690.

27 Scott DA, Klaassens M, Holder AM et al: Genome-wide oligonucleotide-based array comparative genome hybridization analysis of non-isolated congenital diaphragmatic hernia. Hum Mol Genet 2007; 16: 424-430.

28 Excoffon KJ, Avenarius MR, Hansen MR et al: The Coxsackievirus and Adenovirus Receptor: a new adhesion protein in cochlear development. Hear Res 2006; 215: 1-9.

29 Raschperger E, Neve EP, Wernerson A, Hultenby K, Pettersson RF, Majumdar A: The coxsackie and adenovirus receptor (CAR) is required for renal epithelial differentiation within the zebrafish pronephros. Dev Biol 2008; 313: 455-464.

30 Razzaghian HR, Shahi MH, Forsberg LA et al: Somatic mosaicism for chromosome $X$ and $Y$ aneuploidies in monozygotic twins heterozygous for sickle cell disease mutation. Am J Med Genet 2010; 152A: 2595-2598.

31 Hasler R, Begun A, Freitag-Wolf S et al: Genetic control of global gene expression levels in the intestinal mucosa: a human twin study. Physiol Genomics 2009; 38: 73-79.

32 Notini AJ, Craig JM, White SJ: Copy number variation and mosaicism. Cytogenet Genome Res 2008; 123: 270-277.

33 Yurov YB, lourov IY, Vorsanova SG et al: Aneuploidy and confined chromosomal mosaicism in the developing human brain. PloS One 2007; 2: e558.

34 Piotrowski A, Bruder CE, Andersson R et al: Somatic mosaicism for copy number variation in differentiated human tissues. Hum Mutat 2008; 29: 1118-1124.

35 De S.: Somatic mosaicism in healthy human tissues. Trends Genet 2011; 27: 217-223.

36 Mathew SJ, Hansen JM, Merrell AJ et al: Connective tissue fibroblasts and Tcf4 regulate myogenesis. Development 2011; 138: 371-384.

37 Hansson O, Zhou Y, Renström E et al: Molecular function of TCF7L2: Consequences of TCF7L2 splicing for molecular function and risk for type 2 diabetes. Curr Diab Rep 2010; 10 (6): 444-451.

38 Nakamura F, Heikkinen O, Pentikainen OT et al: Molecular basis of filamin A-FilGAP interaction and its impairment in congenital disorders associated with filamin A mutations. PLoS One 2009; 4: e4928.

39 Su ZJ, Hahn CN, Goodall GJ et al: A vascular cell-restricted RhoGAP, p73RhoGAP, is a key regulator of angiogenesis. Proc Natl Acad Sci USA 2004; 101: 12212-12217.

40 Girirajan S, Rosenfeld JA, Cooper GM et al: A recurrent 16p12.1 microdeletion supports a two-hit model for severe developmental delay. Nat Genet 2010; 42: 203-209. 